\title{
Transurethral Plasma Cutting and Prostate Tear in Addition to the Treatment of Hyperplasia of Prostate the Clinical Efficacy of Comparison
}

\author{
Fan-ping $\mathrm{MENG}^{1, \mathrm{a}}$, Di ZOU ${ }^{2}$, Yan $\mathrm{HE}^{2}$ and Ji GAO ${ }^{1, \mathrm{~b},{ }^{*}}$ \\ ${ }^{1}$ Department of Urology, China-Japan Union Hospital, Jilin University, \\ Changchun, Jilin, China \\ ${ }^{2}$ Department of Hepatology, Jilin Academy of Traditional Chinese Medicine, \\ Changchun, Jilin, China \\ a947410581@qq.com, b1217760@qq.com \\ ${ }^{*}$ Corresponding author
}

Keywords: Prostatic hyperplasia, Bipolar plasmakinetic, Resection of prostate, Enucleation of prostate.

\begin{abstract}
Objective To compare the efficacy and complications between bipolar plasmakinetic resection of prostate and bipolar plasmakineticenucleation of prostate for benign prostatic hyperplasia(BPH). Methods 303 cases of BPH were divided into 2 groups, 143 cases aged $(73 \pm 13.4)$ yearswith prostate volume of $(76.5 \pm 22.5) \mathrm{ml}$ underwent PKRP; 160 cases aged $(74 \pm 11.6)$ years with prostate volume of (74.3 \pm 26.5$) \mathrm{ml}$ underwent PKEP.The operative time,resected tissue weighed, blood loss, catheterization time, hospital stay and complications were compared between the 2groups.Results In 2 group, the postoperative RUV, IPSS, QOL and Qmax were significantly improved after both procedures $(\mathrm{P}<0.05)$, but there was nosignificant difference in the above parameters between the 2 groups $(\mathrm{P}>0.05)$.Blood loss, resected tissue weighed were significant differences betweenthe 2 groups $(\mathrm{P}<0.05)$. The operative time, contemporary incontinence and 3 monthes after operation urethral stricture were no significant differencesbetween the 2 groups $(\mathrm{P}>0.05)$. Conclusion The 2 surgical procedures have significant efficacy in the treatment of BPH. But the blood loss andincidences of contemporary incontinence of PKER have more superiority than those of PKRP, so PKER deserves our application.
\end{abstract}

\section{Introduction}

Prostatic hyperplasia (BPH) is the most common diseases of urology, eventually Cause urinary retention, serious damage to the patients quality of life, transurethral resection of the prostate treatment Hyperplasia of prostate can obtain satisfactory curative effect, small trauma, less bleeding and postoperative recovery[1] .The curative effect is satisfied, etc and is widely applied, according to the equipment and the removal of different ways, Transurethral resection of the prostate including transurethral prostatic electricity cut method (TURP), via the urine Electrical cut method in the vaporization of prostate (TUVP) and transurethral bipolar plasma resection of the prostate Plasma prostate (PKRP) and transurethral bipolar carved in addition to surgery (PKEP) and other parties Method. PKRP, PREP is the rise in recent years, developed from the TURP in the treatment of BPH New method, which use plasma bipolar electric knife, not in the body form the current loop, but To use saline wash, more traditional TURP safer, now widely clinically With, but with a plasma between the two kinds of 
operation for electric knife is still lack of comparative study, this research Compare two kinds of operative methods, aims to further analyze the advantages and disadvantages of both, for clinical work as to provide theoretical basis.

\section{Data and Methods}

- Clinical data from January 2008 to December 2008, our hospital of BPH Patients 303 cases, of which 143 cases were treated (PKRP group (PKRP), 160 cases PKER treatment (PKEP group). All patients duration of $2 \sim 21$ years, to some extent Symptoms of urinary tract obstruction.In acute urinary reservoir of joining 250 cases, of which 100 cases happened 2 times More acute urinary retention; Pubis on 6 cases of bladder fistula maker; 98 cases with hypertension; Coronary atherosclerosis heart disease 16 cases; Chronic bronchitis, emphysema, 17 cases; abdomen Ditch hernia 9 cases; Renal insufficiency in 5 cases. 38 cases of diabetes. All patients with preoperative line of rectumDre, after abdominal B to exceed examination determination of prostate volume, residual urine volume (RUV).

- Surgical method Application of Gyrus plasma cutting system, electricity cut $160 \mathrm{w}, 80 \mathrm{w}$ electric coagulation, the conventional TV monitor. Using physiological saline as intraoperative work interface Quality and rinses do not need to be negative. Continuous epidural anesthesia or general anesthesia, do not do the bladder Patients with fistula, take cut stone, transurethral insert F27 electricity cut mirror, in turn, urethral stricture, verumontanum, The prostate, understand the bladder lesions, bilateral ureteral orifice location and bladder neck until fine Mound in the distance, as well as each lobe hyperplasia of prostate PKRP group first selected verumontanum distal logo, 6 points to the bladder neck cut out Ditch, proximal began in the neck and distal end verumontanum near side, depth to the envelope, cutting in turn Right lobe, left lobe and neck glands, 12 points of failure finally make verumontanum near nap, suck out Bladder prostate clastic and bleeding. After coated, electricity cut mirror sheath will the prostate and blunt dissection to the bladder neck, again On both sides of the leaf of the prostate and verumontanum incision to stripping out around the middle of the coated surface, rapid cutting In addition to the prostate tissue, blunt dissection of the hyperplasia of electricity cut mirror sheath to the bladder neck, lateral lobes In case of fiber bundle to separate cutting ring cut off; Before near the bladder neck at 4 o 'clock and eight o 'clock Column gland temporarily not be totally removed.12 o 'clock position after electricity cut method to remove the entire front row The front of the gland is divided into two and a half, pushed to the bladder neck and chopped. Finally the bladder neck and verumontanum on both sides of the tip of the prostate of distal not smooth remaining mucosa, suck out bladder prostate and bleeding.

- Statistical methods, using SPSS 10.0 to deal with data, group than in the group Former statistically difference, achieved the purpose of clinical treatment, is a relatively safe, there is The $t$ test, the comparison of two groups of complications by X-ray 2 Inspection.

\section{The Results}

Patients with two groups of operation time, intraoperative blood loss, removing tissue volume are shown in table 1.art The amount of blood loss, removing tissue, comparing the two groups with statistical difference $(\mathrm{P}<0.05)$.surgery Time no statistical 
difference $(\mathrm{P}>0.05)$. Gouge out in addition to the operative time, blood loss, resection group were better than the control group.

\section{Discussion}

$\mathrm{BPH}$ is a common disease in older men, of urological surgical patients in our country $16.1 \%$, the serious influence the patient's quality of life.Surgical treatment is the most effective Methods. TURP is considered the gold standard for the treatment of BPH Electricity, TURP work PKRP resection of the prostate, it is by the prostatic urethra cavity to prostate surgery coating line Step by step a resection, prostate tissue blood supply is rich, to remove larger when the prostate blood apparent, Significantly increased intraoperative blood loss, ooze blood are too blurry vision, increase operation difficulty, extended Operation time [2]. Because of prostate tissue similar spherical, from the urethra to the outside to remove hyperplasia of prostate Glands, it is difficult to ensure complete removal of hyperplasia of prostate tissue; Prostate distal margin against urine The external sphincter, easy cause urethral sphincter surgery injury postoperative urinary incontinence for a long time, Affect patients with surgery and quality of life, regular to verumontanum near side edge marks the tip of the prostate, The larger lateral lobes prostate tissue significantly more than verumontanum near side, trim the tip of the prostate, In order to avoid damage caused by urethral sphincter long-term postoperative incontinence, may remain close to the Of urethral sphincter lateral lobes glands, leading to a smaller amount of surgically resected specimens, in this study The surgical removal of the specimen weight, on average, only half of the preoperative glands estimated weight [3].

PKEP combined with transurethral surgery and the characteristics of open prostatectomy Cutting loops or sheath by the cavity mirror along the hyperplasia of prostate surgery directly coated layer stripping organization, really Is reached the surgeon removed completely coated in prostate hyperplasia tissue effect, reduced The possibility of residual gland hyperplasia again, removing tissue obviously than PKRP;At the same time, by When stripping to block the blood vessels supplying the hyperplasia gland, retain only the bladder neck a small amount of prostate, Do hemorrhage decreased significantly when the harvest, excision of large, rapid proliferating glands, no Cut through coated with worry, compared with PKRP total operation time is not extended, especially in Larger prostate easier to peel, gland volume increase will not increase significantly For some high-risk patients can control the operation time, operation time, reduce the complications Happen; Through to the surgical coated on residual nodules or further repair of the wound The whole, so that the cavity operation reach or exceed the effects of open surgery; Tear in addition to the prostate, pointed Department is made up of blunt prostate surgery psuedocapsule clearance for mechanical operation, not only completely Removed the tip of the prostate tissue hyperplasia, and avoids the urethral sphincter electricity, Thermal effect damage, prevent the damage caused by a permanent incontinence urethral sphincter ${ }^{[4]}$. The following line should avoid PKEP: (1) the mild hyperplasia of prostate and fibrous tissue Birth is given priority to, these patients with hyperplasia of prostate and surgery coating adhesion obviously not easy separation; (2) the prostatic hyperplasia combined past history of prostatitis, the prostate gland repeatedly parts injection Or radiofrequency treatment; Invasive prostate cancer (3) lack of surgical coated line, cannot do push Cutting action. 


\section{Conclusions}

Compared with PKRP PKEP between the two groups in surgically resected specimens and intraoperative blood loss Statistical significant difference, is obviously better than the PKEP PKRP, is a worthy of promotion The technology.

\section{References}

[1] Transurethral Enucleation and Resection of Prostate in Patients With Benign Prostatic Hyperplasia by Plasma Kinetics [J] . Chunxiao Liu, Shaobo Zheng, Hulin Li, Kai Xu. The Journal of Urology. 2010 (6)

[2] Transurethral resection of prostate: technical progress and clinical experience using the bipolar Gyrus plasmakinetic tissue management system [J] . Gianni Martis,Antonio Cardi,Diana Massimo,Maurizio Ombres,Bruno Mastrangeli. Surgical Endoscopy . 2008 (9)

[3] Bipolar Transurethral Resection in Saline System versus Traditional Monopolar Resection System in Treating Large-Volume Benign Prostatic Hyperplasia. Qi Chen, Li Zhang, Yu Jun Liu, Ji Dong Lu, Guo Ming Wang. Urologia Internationalis. 2009

[4] E-TURP: Technical Evolution of TURP. BREDA G, CELIA A, ZECCOLINI G, et al. European Urology . 2008 